\title{
Synchronization of Delayed Quantum Dot Light Emitting Diodes
}

\author{
HUSSEIN B. AL HUSSEINI ${ }^{1,2}$ \\ ${ }^{1}$ Department of Physics, College of Science, University of Thi Qar, IRAQ \\ ${ }^{2}$ Nassiriya Nanotechnology Research Laboratory (NNRL), Science College, University of Thi Qar. IRAQ
}

\begin{abstract}
Chaos synchronization of two quantum dot light emitting diodes (QDLEDs) theoretically is studied, which is delay coupled via a closed or open -loop and mutual coupling system. Whereas the synchronized- chaotic systems, the dynamics of there are identical to uncoupled DLED under optical feedback effect. Complete synchronization was obtained under certain conditions for the coupling parameters. We evaluated the range of the QDLED's chaos with extrinsic optical feedback in methods of the chaos synchronization residue diagram and discussion as well of the coherence for the optimal coupling strength range. With proper conditions of the coupling parameters and the evaluation methods, the synchronization was satisfactorily obtained between the transmitter and receiver.
\end{abstract}

Key-Words: - QDLEDs, chaos synchronization residue, coherence, control.

Received: May 18, 2020. Revised: November 17, 2020. Accepted: December 7, 2020. Published: December 31, 2020.

\section{Introduction}

Control of complex dynamics within the past years has promoted as one of the major issues in applied nonlinear science [1]. Pioneer progress has been made in neuroscience, inter alia other areas, by widening, methods of chaos control, especially in time-delayed feedback, [2] this phenomenon occurs naturally in many biological systems, including neural networks, in which time delays are caused by delaying proliferation in neurons. [3-5]. Furthermore, delayed feedback loops are intentionally implemented for the purpose of controlling neurological disorders, for example, to get rid of unwanted synchronization in neural networks or to search for symmetrical behavior in communication [6-9]. Here, coupled delayed for two chaotic systems under optical feedback effect were studied. When complete optical coupling occurs in two QDLEDs, the effects of delay can not only be considered important, but the effects of coupling of both electric fields through phase coherence play an important role. Phase coupling of incoming signals may product the constructive or the destructive interference. When the signals synchronization, interference occur in cases where the coupling distance is much greater than the length of the beam cohesion. In this paper, the rate equations model of QDLED with the optical feedback is introduced. Next, is devoted to two estimate of the chaos synchronization and we investigated for different coupling schemes of the feedback. Then, we conclude with discuss the conditions of the coupling parameters for enhancing the behavior of the two QDLED systems, and the chaos synchronization between them.

\section{QDLED Optically feedback model}

For purpose the mode to describe the dynamics of QDLED with optical feedback, as we have a two-part device, the first consists of a spontaneous emission section that contains layers of self-organizing quantum dots( QDs )as an effective layer, and another section that represents a feedback by mirror to reflect light into the active layer. A model describing the spontaneous emission of QDLED has been 
suggested by a microscope-based modified equation system as described in our most recent work. This model allows the treatment of electron transmission in layer of QDs as well as in the surrounding wetting layer (WL) as described by the energy diagram in Fig. 1 .

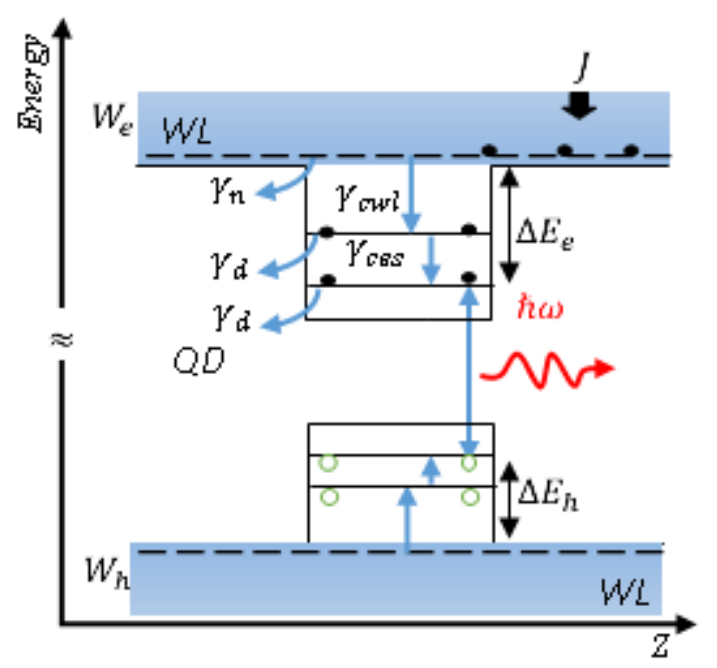

Fig. 1: The energies levels diagram shows the carriers transition and accompanying operations within the active QD LED layer.

In addition, when reformulating the model of rate equations in the case of the presence of optical feedback, it is necessary to takes into account the phase of the electric field. Consequently, the complex field equation was separated from the photons number equation and the phase equation, as this model has been shown to describe many complex phenomena well, including periodic, pulsed, and irregular behavior, as well as complex bifurcation diagrams. The derivation and approximation necessary for our model can be used with LangKobayashi [10] by:

$\frac{d E}{d t}=-\frac{1}{2}(1+i \alpha) d E(t)-\Gamma \frac{E(t)}{2}+\frac{K}{2} E_{\tau} e^{-i \Theta}+E_{S p}(t)$ ....(1)

here $E(t)=\sqrt{S} e^{-i \Phi(t)}$ is the amplitude of the complex normalized slowly varying electric field which given by the photon number $\mathrm{S}$ and the phase $\Phi$ in polar coordinates. Other parameters can be included in the following table:

Table (1): parameters definition listed in Eq. (1).

\begin{tabular}{|c|c|}
\hline$\alpha$ & Linewidth enhancement factor \\
\hline$\omega_{o}$ & $\begin{array}{l}\text { Solitary optical mode angular } \\
\text { frequency }\end{array}$ \\
\hline$K$ & Measures the injected field strength \\
\hline$\tau=2 l / c$ & $\begin{array}{l}\text { Delay time in external cavity for } \\
\text { distance } 1 \text { of mirror }\end{array}$ \\
\hline$\Theta=\omega_{o} \tau$ & $\begin{array}{l}\text { The phase shift of the light during one } \\
\text { round trip in the external cavity }\end{array}$ \\
\hline $\mathrm{c}$ & Speed of light. \\
\hline$E_{\tau}$ & $\begin{array}{l}\text { Electric field amplitude at the delayed } \\
\text { time }(\mathrm{t}-\tau)\end{array}$ \\
\hline$\phi_{\tau}$ & $\begin{array}{l}\text { Optical phase taken at the delayed time } \\
(\mathrm{t}-\tau) \text {. }\end{array}$ \\
\hline
\end{tabular}

The relation of field has of $\left\langle E_{s p}(t) E^{*}(t)\right\rangle=R_{s p} / 2 \quad[11]$ where $\quad E_{\mathrm{sp}}(\mathrm{t})$ corresponding with the zero-mean random field for the stochastic function. The term $\mathrm{R}_{\mathrm{sp}}$ in the photon number equation is used for the effect of spontaneous emission and is given by [12]

$R_{s p}=A n$ and $A=\frac{\beta F(\omega)}{V \rho(\omega)}$

Where

$\beta$ : spontaneous emission rate;

$\mathrm{V}$ : normalization volume;

$\mathrm{F}(\mathrm{w})$ : normalized lineshape function;

$\rho(w)$ :density of photon states for the uniform QD.

Also, when investigating the causes of chaos and instability in non-linear optical systems, treatment of deterministic values becomes 
possible in the light of verification in statistical processes. The goal is to transform the complex stochastic differential equation (SDE) for $E(t)$ into two real SDE for the photon number $S(t)$ and the phase of electric field $\phi(t)$. Neglecting the stochastic term this is just a transformation to polar coordinates, after that adding stochastic averages after completing the polar formula conversion process, the complex field equation becomes in terms of the number of photons and the phase of the electric field, in addition to the rate equations for the number of electrons in the QDs $\left(n_{Q D}\right)$ and in the WL $\left(n_{w l}\right)$ read:

$\frac{d S}{d t}=-d S-\Gamma S+A n+K \sqrt{S S_{\tau}} \cos \left(\phi-\phi_{\tau}+w_{o} \tau\right)$

$\frac{d \Phi}{d t}=\frac{1}{2} \alpha d-K \sqrt{S_{\tau} / S} \sin \left(\phi-\phi_{\tau}+w_{o} \tau\right)$

$\frac{d n_{Q D}}{d t}=\gamma_{c} n_{w l}\left(1-\frac{n_{Q D}}{2 N_{d}}\right)-\gamma_{r} n_{Q D}-(A n-d S)$

$\frac{d n_{w l}}{d t}=\frac{J}{e}-\gamma_{n} n_{w l}-\gamma_{c} n_{w l}\left(1-\frac{n_{Q D}}{2 N_{d}}\right)$

here, $A$ is the rate of spontaneous emission in the optical mode. The parameters $d, \Gamma$ are the absorption and output coupling rate of photons, $\gamma_{r}$ and $\gamma_{n}$ are the non-radiative decay rates of the number of carriers in the upper levels and WL respectively; $N_{d}$ is the is the total number of QDs; and $J$ is the injection current, $e$ is electron charge, $\gamma_{c}$ is the capture rate from WL into an empty quantum dot.

The transition is homogeneously broadened for a three-level semi atomic system, where one can get from Einstein's equation [14]

$d=A n_{o}$

here $n_{o}$ is the occupation number of the QD layer. In such a case, for the spontaneous emission coefficient and absorption coefficient have similar trajectory. For organic or semiconductor emitters, as realistic material systems, there remains the possibility of inhomogeneous broadened in the upper and lower levels. In order to establish a valid formula between the absorption and the spontaneous emission spectrum must be taken into account the population distributions at the lower and higher levels [15].

In the case of coupling with a self-optical feedback, the time-dependent phase has an important role because of the interaction of the phase with other variables of the signal and the medium. We can investigate numerically solving of the dynamics of QDLED under optical feedback by using the above equations. In [16], a solitary frequency rate equation, affective phase does not on the other variables and, thus, it is only described by the photon and carrier number rate equations. Nevertheless, it is not possible to cancel the role a time development of the phase under the influence of the coupled field, because the phase is associated with the presence of optical feeding, as is evident from two equations of the phase and the number of photons above.

\section{Chaos synchronization of delay-coupled QD LED}

We investigate theoretically in this section the examiner for chaos synchronization in the two QD LEDs which are coupling together and additionally each one received self- optical feedback. In Fig. 2 the basic coupling scheme is depicted. The rate equation mentioned in the previous section can be rewritten according to the coupling methods shown in the above figure for both the transmitter and receiver by adding the light transmission terms. 
(a) Closed-loop system

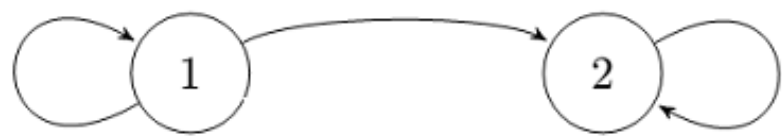

(b) Open -loop system

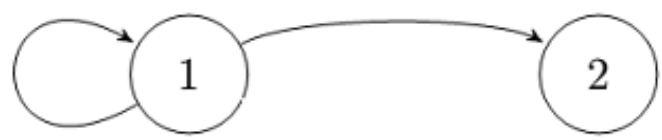

(c) Mutual coupling system

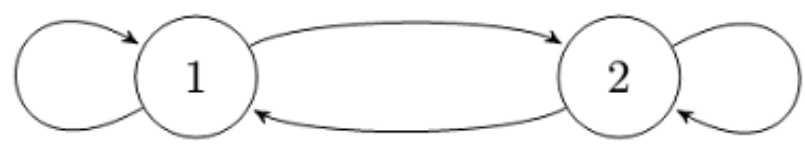

Fig. 2: Diagram of synchronization methods for two coupling chaotic systems under optical feedback. (a) Close loop coupling system, (b) open loop coupling system, and (c) mutual coupling system. 1: transmitter QDLED, 2: receiver QDLED.

The filed equations for the transmitter and the receiver QD LED read

$$
\begin{aligned}
\frac{d S_{1}}{d t}=- & d_{1} S_{1}-\Gamma_{1} S_{1}+A_{1} n_{1}+K_{1} \sqrt{S_{1} S_{\tau 1}} * \\
& \cos \left(\phi_{1}-\phi_{\tau 1}+w_{o 1} \tau_{1}\right) \\
\frac{d \Phi_{1}}{d t}= & \frac{1}{2} \alpha_{1} d_{1}-K_{1} \sqrt{S_{\tau 1} / S_{1}} \sin \left(\phi_{1}-\phi_{\tau 1}+w_{o 1} \tau_{1}\right)
\end{aligned}
$$

where subscript 1 refers here to transmitter number 1 . The rate equations for the receiver are written as follows

$$
\begin{aligned}
& \text { are written as follows } \\
& \qquad \begin{array}{l}
\frac{d S_{2}}{d t}=-d_{2} S_{2}-\Gamma_{2} S_{2}+A_{2} n_{2}+K_{2} \sqrt{S_{2} S_{\tau 2}} \cos \left(\phi_{2}-\phi_{\tau 2}\right. \\
d t
\end{array}=\frac{1}{2} \alpha_{2} d_{2}-K_{1} \sqrt{S_{\tau c} / S_{1}} \text { * } \\
& \left.\quad+w_{o 2} \tau_{2}\right)+K_{12} \sqrt{S_{1} S_{\tau c}} \cos \left(\phi_{1}-\phi_{\tau c}+\phi_{o 1} \tau_{c}+\Delta w t\right)
\end{aligned}
$$

$$
\begin{aligned}
\frac{d \Phi_{2}}{d t} & =\frac{1}{2} \alpha_{2} d_{2}-K_{2} \sqrt{S_{\tau 2} / S_{2}} \sin \left(\phi_{2}-\phi_{\tau 2}+w_{o 2} \tau_{2}\right) \\
& -K_{12} \sqrt{S_{\tau c} / S_{1}} \sin \left(\phi_{1}-\phi_{\tau c}+w_{o 1} \tau_{c}+\Delta w t\right)
\end{aligned}
$$

Here $\tau_{c}$ is delay-coupled systems with $\tau$ is delay-time of self-optical feedback. $K_{i j}$ is a coupling strength and $\emptyset_{\mathrm{ij}}$ is a coupling phase of each connection. Where subscript 2 denotes the receiver QDLED, and $\Delta \omega=\omega_{1}-\omega_{2}$ represents to detuning of the angular frequency between the two coupled QDLEDs. The last terms in the last equations express the effect of the pairing with the signal sent by the transmitter. When openloop case the self-optical feedback strength is zero in the receiver system, $\left(\mathrm{k}_{2}=0\right)$, as in Fig. 2.b.

In Fig. 2.c we discuss chaos synchronization in mutually coupled QDLED modeled. Although the QDLED plays a role for the external mirror of the counterpart QDLED even without self optical feeding, systems can show an increase in additional perturbation due to mutual coupling. The rate equations for a QDLED transmitter are written by

$$
\begin{gathered}
\frac{d S_{1}}{d t}=-d_{1} S_{1}-\Gamma_{1} S_{1}+A_{1} n_{1}+K_{2} \sqrt{S_{2} S_{\tau c}} * \\
\cos \left(\phi_{1}-\phi_{\tau c}+w_{o 1} \tau_{1}+\Delta w t\right) \\
\frac{d \Phi_{1}}{d t}=\frac{1}{2} \alpha_{1} d_{1}-K_{2} \sqrt{S_{\tau c} / S_{2}} * \\
\sin \left(\phi_{1}-\phi_{\tau c}+w_{o 1} \tau_{1}+\Delta w t\right)
\end{gathered}
$$

Similarly, the receiver QLED rate equations are given by form as

$$
\begin{gathered}
\frac{d S_{2}}{d t}=-d_{2} S_{2}-\Gamma_{2} S_{2}+A_{2} n_{2}+K_{1} \sqrt{S_{1} S_{\tau c}} *(8 . \mathrm{a}) \\
\cos \left(\phi_{2}-\phi_{\tau c}+w_{o 2} \tau_{2}-\Delta w t\right)
\end{gathered}
$$

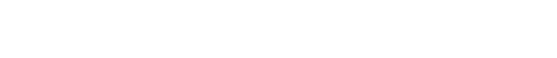


In the three methods above of the coupling systems, there are two control ways of chaos synchronization: one is based on optical fed back as signal amplification phenomena and the second is complete chaotic synchronization. With two systems coupling, one QDLEDs playing the master role and the other being a slave, the residue of chaos synchronization is defined with the following equation:

$R_{\text {chaos }}=\left|\frac{S_{1}-S_{2}}{S_{1}}\right|$

Where $S_{1}$ and $S_{2}$ are the intensities maximum of the transmitter and receiver. The effect of parameter asymmetry, especially the coupling strength between both the transmitter and receiver, is extremely important in applications for securing encrypted communications.

The diagram of the chaos synchronization residue is depicted in Fig. 3, where 0 expresses the complete synchronization between the transmitter and receiver parameters. Fig. 3 (a) shows the residue of unidirectional coupling systems chaos synchronization. The acceptable residues for the parameter mismatches are large in closed-loop coupling systems chaos synchronization and we can expect asymmetry chaos synchronization. From figure, the chaos residues are always smaller than 0.5 of the average QDLED intensity variations for three values of delayed-time $0.07,0.37$ and 0.67 respectively, because of the synchronization comes from signal amplification phenomena due to optical feedback, while asynchronous waveform comes from the transmitter signal. The subplot illustration is for case 0.67 .

By continuing to investigate the consistency of the transmitter and receiver signals, we note the similarity between them when the residual match of the signals varies by a few percent. Complete synchronization is achieved with high accuracy when matching the parameters as in Fig. 3(b) and at the same value of delayed-time in the previous figure, and the residue of synchronization increases rapidly with increasing parameter of the strength of coupling. The best symmetry of synchronization residues is also seen in the open-loop coupling system. Hence, there is a need to set strict conditions for the synchronization of successful chaos in the complete synchronization.

From the results mentioned above, in the two delayed-coupled systems, the parameters must be identical to achieve the requirements for complete synchronization of the disorder, but it is desirable to note that there are certain ranges of discrepancies in the parameter mismatches that lead to a slight deterioration in the correlation relationship between the transmitter and receiver outputs. Achieve complete chaos synchronization in mutual coupled system see in Fig.4. It also notes that it is not always possible to get the best synchronization when the feedback strength is zero.

The sudden transition to synchronization condition could also be described by calculating the mean of the coherence value for all oscillators in synchronized and nonsynchronized statuses.

A value of 1 refers to coherence in the case of interdependence of the operations between $\mathrm{x}$ and $\mathrm{y}$ and the coherence takes values close to zero in the absence of any interaction at the frequency $\mathrm{w}$, at certain values of the frequency, if the coherence is equal to 1 , then the synchronization is complete and the two signals coincide at this frequency and counter completely from treatment of synchronization residue in the above results. Conversely, a coherence which is equal to $\mathbf{0}$ suggests that the signals are totally unrelated at the frequency. We can be observed the Interactions in linear stochastic systems employing cross-spectral analysis. For that, the cross-spectrum $P_{x y}(f)$ which is the Fourier transform of the crosscovariance function of processes $\mathrm{x}$ and $\mathrm{y}$, is normalized by the auto-spectra $P_{x x}(f)$ which is the Fourier-transform of the auto-covariance 

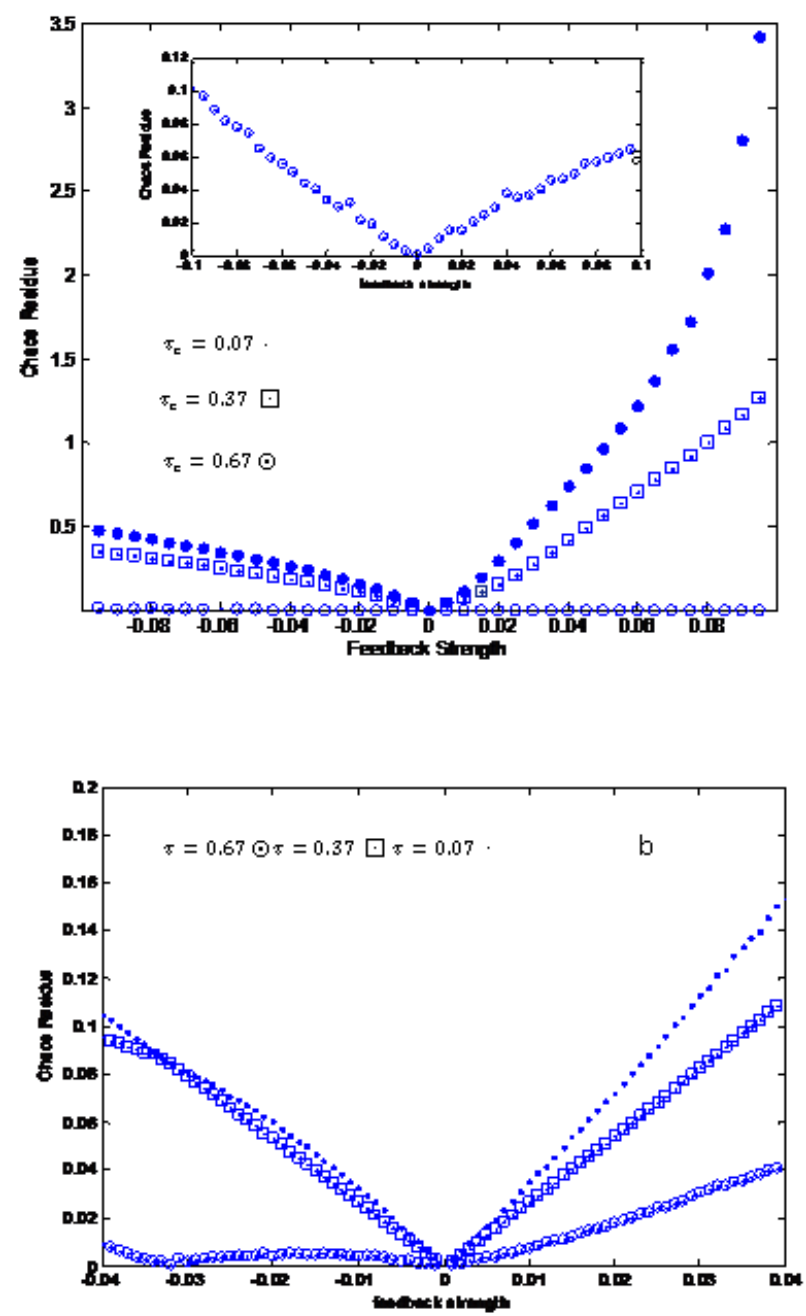

Fig. 3: Calculated chaos synchronization residue as a function of parameter mismatch (coupling strength). $\tau_{c}$ : coupled delayed time. (a) closed-loop system (behavior asymmetry) and (b) open-loop system (behavior symmetry) chaos synchronization. The parameter values are $I_{o}=0.6, k_{1}=k_{2}=6.2 \times 10^{-5}$ and $w_{1}=$ $w_{2}=3.2 \pi$.

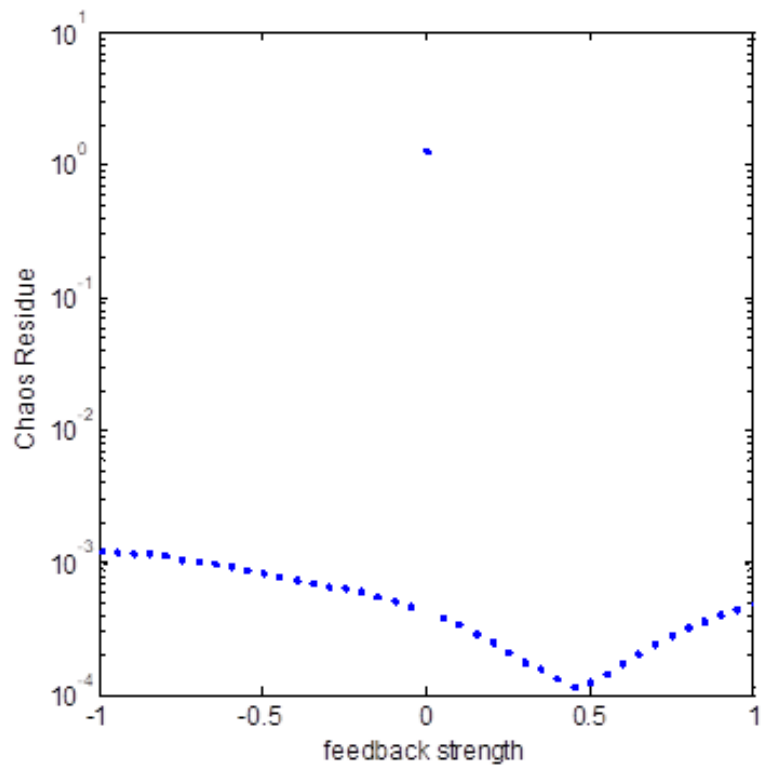

Fig. 4: Calculated chaos synchronization residue as a function of coupling strength. The parameter values are $\tau=40, \tau_{c}=70, I_{o}=5.9$, $k_{1}=6.2 \times 10^{-5}, k_{2}=0$ and $w_{1}=w_{2}=0.2 \pi$.

function of processes $\mathrm{x}$ and $\mathrm{y}$. respectively, leading to the coherence function $(C x y(f))$. The coherence is a function of frequency that measures the degree of linear dependency of two signals by testing whether they contain similar frequency components. It is:

$C_{x y}(f)=\frac{\left|P_{x y}(f)\right|^{2}}{P_{x x}(f) P_{y y}(f)}$

Fig.5 presents the dependence of synchronization quality and the chaotic complexities on the mean complex value. In this condition, the synchronization quality is quantified by the coherence (cross correlation) among the entire oscillators output. The complexities of the chaotic optical systems are indicated by the correlation of the time series of the oscillator's intensities. 

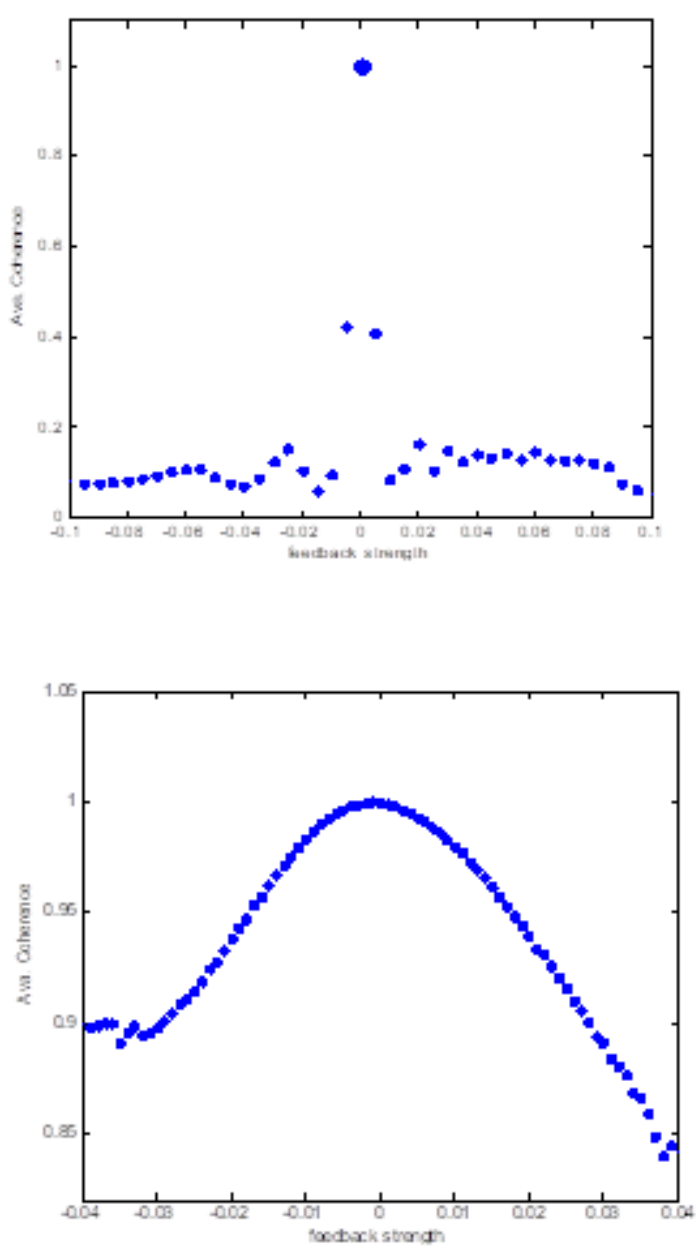

Fig. 5: The mean coherence value $(C)$, when there is no coupling between the two chaotic systems $\left(K_{12}=0\right)$, the mean $C$ value is closed to zero and around 1 for synchronization condition. (a) closed-loop system and (b) openloop system chaos synchronization. The parameter values are $I_{o}=5.6, k_{1}=k_{2}=$ $6.2 \times 10^{-5}$ and $w_{1}=w_{2}=3.2 \pi$.

Generally, the synchronization degradation is attributed to the effect of the nonlinear saturation of the system. Here we explain this phenomenon from the two different perspectives, firstly as shown in Fig. 5 the coherence among the oscillators in low frequency range is much prominent, which indicates that the high frequency range.
At small value of the coupling between the two chaotic systems $\left(K_{12}=0\right)$, high quality synchronization can be presented in overall range of coupling phases considered here.

\section{Conclusion}

In conclusion, chaos synchronization conditions with optical feedback in QDLEDs are discussed. In all methods coupling, the optical coupling phases and the mismatching strengths play a critical role for the signals synchronizability. The condition of the lineup corresponds to specific interference conditions, so the signals of both QDLEDs should interfere in such a way that each QDLED receives the same output signal, relative to its own phase. This agrees to the presence of an identical complete synchronization. During interference, the effective of phases may be similar to mismatches in the coupling strengths. Further, a different kind of measurement the synchronization phenomenon as reported. This method allows measuring the mismatch between the coupled systems. In this work, overall synchronization states were achieved by controlling and adjusting the states of the two coupled systems such as setting delay time and coupling strength while keeping the injection current steady. Moreover, we have realized theoretical synchronization in terms of the chaos synchronization residue and coherence of the response times, measured between interacting QDLEDs units. From both the chaos synchronization residue and coherence have been evaluated what revealed the symmetric and asymmetric increase in its value as the coupling strength was modulated.

\section{References:}

[1] Handbook of Chaos Control, edited by E. Scholl and H. G. Schuster. WileyVCH, Weinheim, 2008.

[2] K. Pyragas, Continuous Control of Chaos by Self-Controlling Feedback, Phys. Lett. A170, 421 .1992.

[3] Balanov, A. G., N. B. Janson and E. Scholl (2004). Control of noise-induced oscillations by delayed feedback. Physica D 199, 1-12.

[4] Fitz Hugh, R. (1960). Thresholds and plateaus in the Hodgkin-Huxley nerve 
equations. J. Gen. Physiol. 43(5), 867896.

[5] Gerstner, W. and W. Kistler (2002). Spiking neuron models. Cambridge University Press. Cambridge.

[6] Haken, H. (2006). Brain Dynamics: Synchronization and Activity Patterns in Pulse-Coupled Neural Nets with Delays and Noise. Springer Verlag $\mathrm{GmbH}$. Berlin.

[7] L. M. Pecora and T. L. Carroll, Phys. Rev. Lett. 64, 821, 1990.

[8] L. O. Chua, C. W. Wu, A. Huang, and G. Q. Zhong, "A universal circuit for studying and generating chaos-Part I: Routes to chaos", IEEE Transaction on Circuits and Systems-1, 40, 10,731-744, 1993.

[9] M. Kennedy "Chaos in the Colpitts oscillator". IEEE Transaction on Circuits and Systems-1, vol.41, pp.771774, 1994.

[10] F. Plenge, P. Rodin, E. Scholl, and K. Krischer, Domain coarsening of stripe patterns close to onset. Phys. Rev. E 64, $056229,2001$.

[11] G. S. Medvedev and J. E. Cisternas, Multimodal regimes in a compartmental model of the dopamine neuron,.Physica D 194, 333,2004 .

[12] H. B. Al Husseini, K. A. Al Naimee, A. H. Al-Khursan, and A. H. Khedir," External modes in quantum dot light emitting diode with filtered optical feedback." Journal of Applied Physics 119, $224301 \quad$ (2016); doi: 10.1063/1.4953651. View online: http://dx.doi.org/10.1063/1.4953651

[13] K. Al-Naimee, F. Marino, M. Ciszak, R. Meucci, and F. T. Arecchi, Chaotic spiking and incomplete homoclinic scenarios in semiconductor lasers with optoelectronic feedback. New J. Phys. 11, 073022, 2009.

[14] K. Al Naimee, H. Al Husseini, S.F. Abdalah, A. Al Khursan, A.H. Khedir, R. Meucci, F.T. Arecchi, "Complex dynamics in Quantum Dot Light Emitting Diodes," Eur. Phys. J. D, 69: 257, 1-5, 2015

[15] K. Al Naimee, H. Al Husseini, S.F. Abdalah, A. Al Khursan, A.H. Khedir, R. Meucci, F.T. Arecchi, "Mixed mode oscillations and chaotic spiking in Quantum Dot Light Emitting Diodes,"
Proceedings of the IEEE $06 / 2014 ; 78$, DOl; 10, 1016/j. chaos, 2015, 07, 033.

[16] H. Al Husseini, A. Al Khursan, K. Al Naimee, S.F. Abdalah, A.H. Khedir, R. Meucci, F.T. Arecchi, "Modulation Response, Mixed mode oscillations and chaotic spiking in Quantum Dot Light Emitting Diodes," ELSEVIER, Chaos, Solitons \& Fractals, Nonlinear Science, and Nonequilibrium and Complex Phenomena, 78, 229-237, 2015.

Acknowledgements. This work is supported by the Nassiriya Nanotechnology Research Laboratory (NNRL), Science College, University of Thi Qar, Iraq.

Compliance with ethical standards

Conflicts of interest. The authors declare that they have no conflict of interest.

\section{Creative Commons Attribution License 4.0 (Attribution 4.0 International, CC BY 4.0)}

This article is published under the terms of the Creative Commons Attribution License 4.0 https://creativecommons.org/licenses/by/4.0/deed.en US 